\title{
Detection of MDM2 gene amplification or protein expression distinguishes sclerosing mesenteritis and retroperitoneal fibrosis from inflammatory well-differentiated liposarcoma
}

\author{
Joshua Weaver ${ }^{1}$, John R Goldblum ${ }^{1}$, Sondra Turner ${ }^{1}$, Raymond R Tubbs ${ }^{1}$, Wei-Lein Wang ${ }^{4}$, \\ Alexander JF Lazar ${ }^{4}$ and Brian P Rubin ${ }^{1,2,3}$ \\ ${ }^{1}$ Department of Anatomic Pathology, Pathology and Laboratory Medicine Institute, The Cleveland Clinic and \\ the Cleveland Clinic Lerner College of Medicine, Case Western Reserve University, Cleveland, OH, USA; \\ ${ }^{2}$ Taussig Cancer Center, Cleveland, OH, USA; ${ }^{3}$ Lerner Research Institute, Cleveland, OH, USA and \\ ${ }^{4}$ Department of Pathology, Sarcoma Research Center, UT-MD Anderson Cancer Center, Houston, TX, USA
}

\begin{abstract}
Inflammatory liposarcoma is a variant of well-differentiated liposarcoma/atypical lipomatous tumor that consists of a mixture of lymphocytes, histiocytes, scattered atypical stromal cells, mature adipocytes, and rarely lipoblasts. When the inflammatory infiltrate predominates, the morphological features overlap with various fibroinflammatory disorders including sclerosing mesenteritis and retroperitoneal fibrosis, making the diagnosis difficult. Well-differentiated liposarcoma/atypical lipomatous tumor and dedifferentiated liposarcoma have characteristic molecular markers in the form of giant marker and ring chromosomes consisting of amplicons of 12q13-15, which includes MDM2. MDM2 immunohistochemistry (IHC) (Zymed; clone IF2) and dual color fluorescence in situ hybridization utilizing MDM2 (12q15) and chromosome 12 centromeric probes were performed on formalin-fixed and paraffin-embedded specimens from inflammatory well-differentiated liposarcoma (17 cases), sclerosing mesenteritis (14 cases), and idiopathic retroperitoneal fibrosis (10 cases). MDM2 expression as detected by IHC is a very sensitive tool in recognizing inflammatory well-differentiated liposarcoma (17 of 17); however, $21 \%$ (3 of 14 ) and $10 \%$ ( 1 of 10$)$ of sclerosing mesenteritis and retroperitoneal fibrosis, respectively, displayed weak MDM2 immunoexpression. The MDM2 fluorescence in situ hybridization assay was very specific for inflammatory well-differentiated liposarcoma as 15 of $17(88 \%)$ cases showed MDM2 amplification, whereas none of the cases of sclerosing mesenteritis or idiopathic retroperitoneal fibrosis showed amplification. Five cases of retroperitoneal fibrosis were noncontributory secondary to autofluorescence, potentially limiting the usefulness of the assay in certain situations such as inappropriate fixation. Increased MDM2 expression and/or MDM2 amplification can be employed to aid discrimination of inflammatory well-differentiated liposarcoma from fibroinflammatory mimics. MDM2 fluorescence in situ hybridization is a very specific method $(100 \%)$, but less sensitive $(88 \%)$, whereas MDM2 expression by IHC is very sensitive $(100 \%)$, but less specific $(83 \%)$. Therefore, a positive screen of difficult cases with MDM2 IHC would require confirmation by the fluorescence in situ hybridization. However, lack of MDM2 immunoexpression would rule out the possibility of inflammatory well-differentiated liposarcoma.
\end{abstract}

Modern Pathology (2009) 22, 66-70; doi:10.1038/modpathol.2008.153; published online 3 October 2008

Keywords: MDM2; FISH; inflammatory well-differentiated liposarcoma; dedifferentiated liposarcoma; atypical lipomatous tumor; sclerosing mesenteritis

Sclerosing mesenteritis is an uncommon idiopathic condition that presents symptoms associated with an abdominal mass. It typically appears as a stellate mass radiographically, which on imaging can be

Correspondence: Dr BP Rubin, MD, PhD, Department of Anatomic Pathology, L25, 9500 Euclid Avenue, Cleveland, OH 44195, USA. E-mail: rubinb2@ccf.org

Received 30 May 2008; revised 20 August 2008; accepted 26 August 2008; published online 3 October 2008 indistinguishable from some primary soft tissue sarcomas or some cases of metastatic carcinoma. ${ }^{1}$ Differentiation of this condition from malignancies is essential because treatment is nonsurgical. Previous reports in the literature have used a variety of terms to describe this lesion including sclerosing mesenteritis, retractile mesenteritis, mesenteric lipodystrophy, and mesenteric panniculitis. ${ }^{2-5}$ The plethora of terminology developed can be explained by the wide spectrum of histologic 
findings including variable amounts of fibrosis, chronic inflammation, and fat necrosis. In a large series of 84 cases, Emory et $a l^{6}$ concluded that all of these entities represented different histologic variants of a single entity with similar clinical features and a variable spectrum of all the three classic histologic findings-fibrosis, chronic inflammation, and fat necrosis, proposing the unifying term sclerosing mesenteritis.

Idiopathic retroperitoneal fibrosis is a rare disorder that results in fibrosis and chronic inflammation of retroperitoneal tissue around a nondilated abdominal aorta with entrapment of adjacent structures such as the ureters and other abdominal organs, resulting in a variety of clinical symptoms. ${ }^{7}$ The histologic appearance of retroperitoneal fibrosis consists of variable proportions of fibroblastic proliferation and mononuclear inflammatory infiltrate, depending on the stage of the lesion. ${ }^{8}$ The diagnosis of idiopathic retroperitoneal fibrosis is typically strongly suggested by radiographic findings. However, biopsy may be necessary when an atypical clinical presentation is encountered or when a neoplastic process is suspected. Medical treatment, normally immunosuppression through corticosteroids, usually results in a favorable outcome. ${ }^{7}$

Well-differentiated liposarcoma/atypical lipomatous tumor is a common soft tissue tumor that frequently presents in the retroperitoneum and abdomen of adults. ${ }^{9}$ These tumors are at risk of progressing to dedifferentiated liposarcoma in this location. Well-differentiated liposarcoma/atypical lipomatous tumor can be subclassified into a variety of subtypes based on the predominant histological pattern, including lipoma-like, sclerosing, spindle cell, and inflammatory subtypes. ${ }^{10}$ The inflammatory subtype of well-differentiated liposarcoma/ atypical lipomatous tumor is a rare variant that most often is located in the retroperitoneum and can be difficult to recognize due to the marked mixed inflammatory infiltrate that obscures the adipocytes and diagnostic atypical cells, particularly in small biopsies. ${ }^{11,12}$

Well-differentiated liposarcoma/atypical lipomatous tumor and dedifferentiated liposarcoma harbor ring and giant marker chromosomes consisting of 12q13-15 amplicons containing several genes, including $M D M 2 .{ }^{13-15}$ Detection of MDM2 expression by immunohistochemistry (IHC) and genetic amplification by fluorescence in situ hybridization (FISH) have been shown to be useful ancillary diagnostic tools to distinguish well-differentiated liposarcoma/ atypical lipomatous tumor from their morphologic mimickers. ${ }^{16,17}$

The inflammatory subtype of well-differentiated liposarcoma/atypical lipomatous tumor is often difficult to distinguish morphologically from sclerosing mesenteritis and idiopathic retroperitoneal fibrosis, especially on needle biopsy specimens when the characteristic atypical cells of welldifferentiated liposarcoma/atypical lipomatous tumor may not have been sampled or are obscured by the brisk inflammatory infiltrate. Herein, we evaluate the utility of IHC and FISH to detect MDM2 expression and MDM2 amplification, respectively, in a series of lesions to determine whether these tools could help differentiate inflammatory welldifferentiated liposarcoma/atypical lipomatous tumor from sclerosing mesenteritis and retroperitoneal fibrosis.

\section{Materials and methods}

In total, 10 cases of retroperitoneal fibrosis, 14 cases of sclerosing mesenteritis, and 17 cases of welldifferentiated liposarcoma inflammatory subtype were obtained from the archives of the Department of Anatomic Pathology at the Cleveland Clinic and UT-MD Anderson Cancer Center. Appropriate institutional review board approval was in place at each institution. Hematoxylin and eosin stained sections of both needle biopsies and resection specimens were independently reviewed by two soft tissue pathologists (JRG and BPR).

Whole-tissue sections of all the cases were utilized to perform MDM2 FISH. The FISH assay was performed with a laboratory-developed BAC label probe cocktail from RP11-775J10 and RP11450G15 BAC DNAs purchased from Roswell Park Cancer Institute, Buffalo, NY, USA, specific for MDM2 (12q15) and a probe specific for the centromeric region of chromosome 12 (Abbott Molecular, DesPlaines, IL, USA) according to an established laboratory protocol, as previously described. ${ }^{18}$ The MDM2 FISH assays were scored blindly by counting a minimum of 40 nuclei per case under oil immersion at $\times 100$ magnification with a DAPI/ Green/Red triple band pass filter. Only nuclei with at least two CEP12 signals were evaluated to minimize nuclear truncation artifact, and overlapping tumor nuclei were also excluded from evaluation to decrease false-positive scoring. The average number of MDM2 and CEP12 signals was then determined and a $M D M 2 / C E P 12$ ratio was calculated for each case. A ratio of $\geq 2.0$ was considered amplified for the MDM2 gene, whereas a ratio of $<2.0$ was considered nonamplified. A ratio of $<2.0$ with $>2$ signals of both probes was considered polysomic for CEP12.

MDM2 immunostaining was performed on 4- $\mu$ mthick whole-tissue paraffin-embedded cut sections on glass slides (Superfrost + ) using a Discovery XT (Ventana Medical Systems/Tucson, AZ, USA) automated IHC instrument with a biotin-free, multimer technology detection kit and conjugate (ChromoMap DAB Kit (760-159)/OmniMap anti-Ms HRP (7604310), Ventana). CC1 (950-124, Ventana) was used for antigen retrieval and the antibody was incubated for $1 \mathrm{~h}$ at room temperature. The primary antibody used was from Zymed Laboratories for MDM2 (clone IF2, dilution 1:50). 
Immunostained slides were evaluated by the two independent soft tissue pathologists (JRG and BPR), discordant cases were reevaluated collegially. MDM2 expression by IHC was scored based on percentage of lesional nuclei staining positive: $0=0 \%, 1+=1-25 \%, 2+=26-50 \%$, and $3+$ $=>50 \%$. A tumor was considered MDM2 positive when a score of $\geq 1+$ was assigned. Non-nuclear cytoplasmic staining was not interpreted as positive.

\section{Results}

MDM2 protein as detected by IHC was found in 17 of $17(100 \%)$ inflammatory well-differentiated liposarcomas, 1 of $10(10 \%)$ cases of idiopathic retroperitoneal fibrosis, and 3 of $14(21 \%)$ cases of sclerosing mesenteritis. A follow-up biopsy was available from the single patient with retroperitoneal fibrosis with MDM2 expression by IHC and their second biopsy was negative (0) for MDM2. None of the three patients with sclerosing mesenter- itis who had MDM2 positivity had follow-up pathology specimens in our archives. The MDM2 expressions were all $1+(1-25 \%)$ in the cases of benign fibroinflammatory disorders and $2+(25-$ $50 \%)$ or $3+(>50 \%)$ in 9 out of 17 cases $(52.9 \%)$ of liposarcoma (Figure 1). Aberrant or nonspecific MDM2 immunostaining was present in other cell types including the cytoplasm of fibroblasts in one case of retroperitoneal fibrosis, the cytoplasm of plasma cells in another case of retroperitoneal fibrosis, and nuclear staining of histiocytes in two cases of sclerosing mesenteritis.

MDM2 amplification was present in 15 of 17 cases of inflammatory well-differentiated liposarcoma/ atypical lipomatous tumor and absent in all cases of sclerosing mesenteritis $(n=14)$ and retroperitoneal fibrosis $(n=5)$, with appropriate signals (Figure 2). The remaining five cases of retroperitoneal fibrosis displayed unsatisfactory MDM2 FISH results due to autofluorescence. The average MDM2/ CEP12 ratio of inflammatory well-differentiated liposarcoma/atypical lipomatous tumor was 5.35,

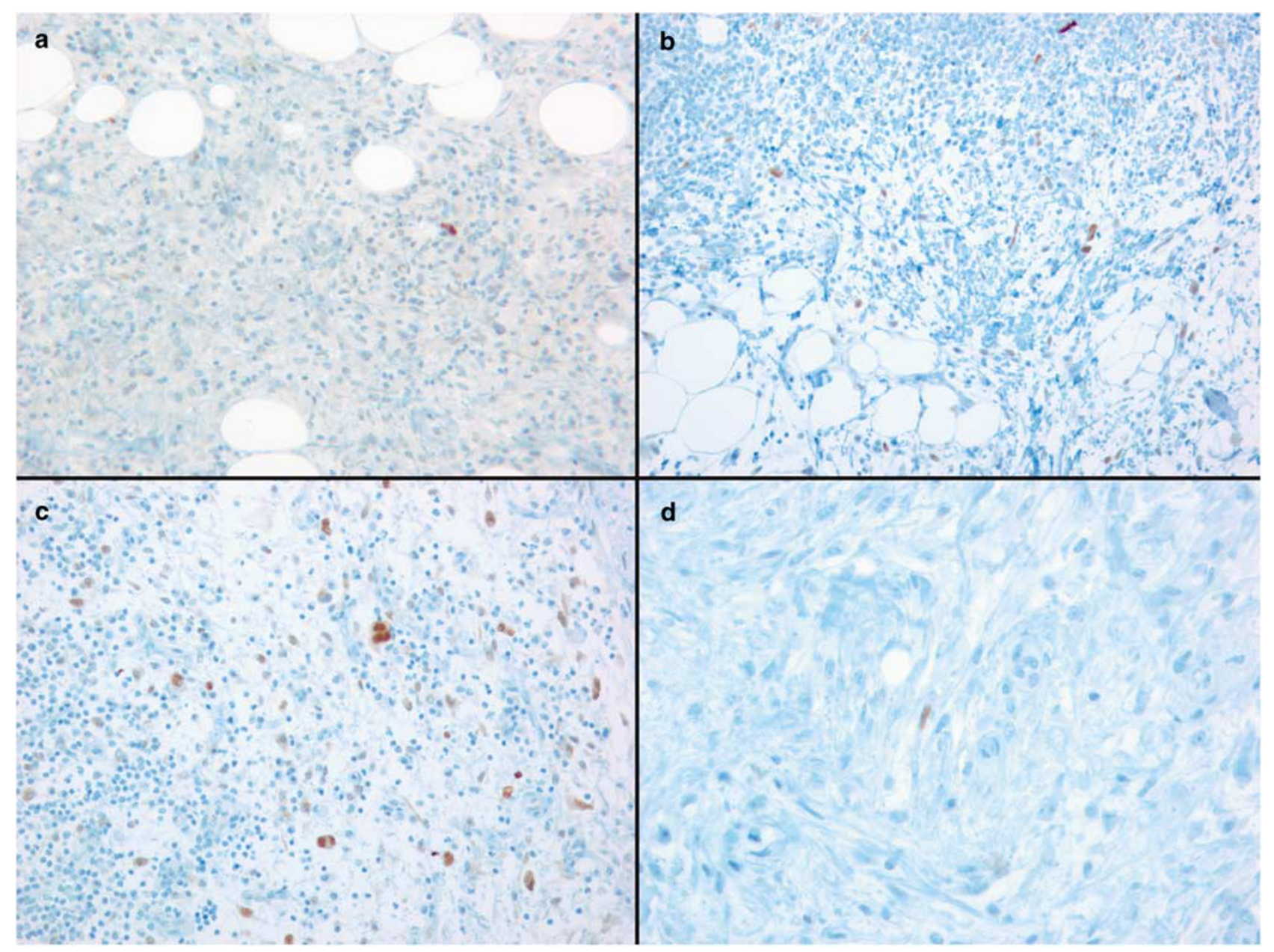

Figure 1 Range of immunohistochemical (IHC) staining for MDM2 observed: (a) 1+ MDM2 immunoexpression (1-25\% positivity lesional cells) in a case of inflammatory well-differentiated liposarcoma/atypical lipomatous tumor (WDLS/ALT) (MDM2 IHC, $\times 20),(\mathbf{b})$ $2+$ MDM2 immunoexpression (26-50\% positivity lesional cells) within an example of inflammatory WDLS/ALT (MDM2 IHC, $\times 20$ ), (c) $3+$ MDM2 immunoexpression ( $>50 \%$ positivity lesional cells) within an inflammatory WDLS/ALT (MDM2 IHC, $\times 20)$, and (d) focal, 1 + MDM2 immunoexpression (1-25\% positivity lesional cells) in a case of sclerosing mesenteritis (MDM2 IHC, $\times 40$ ). 

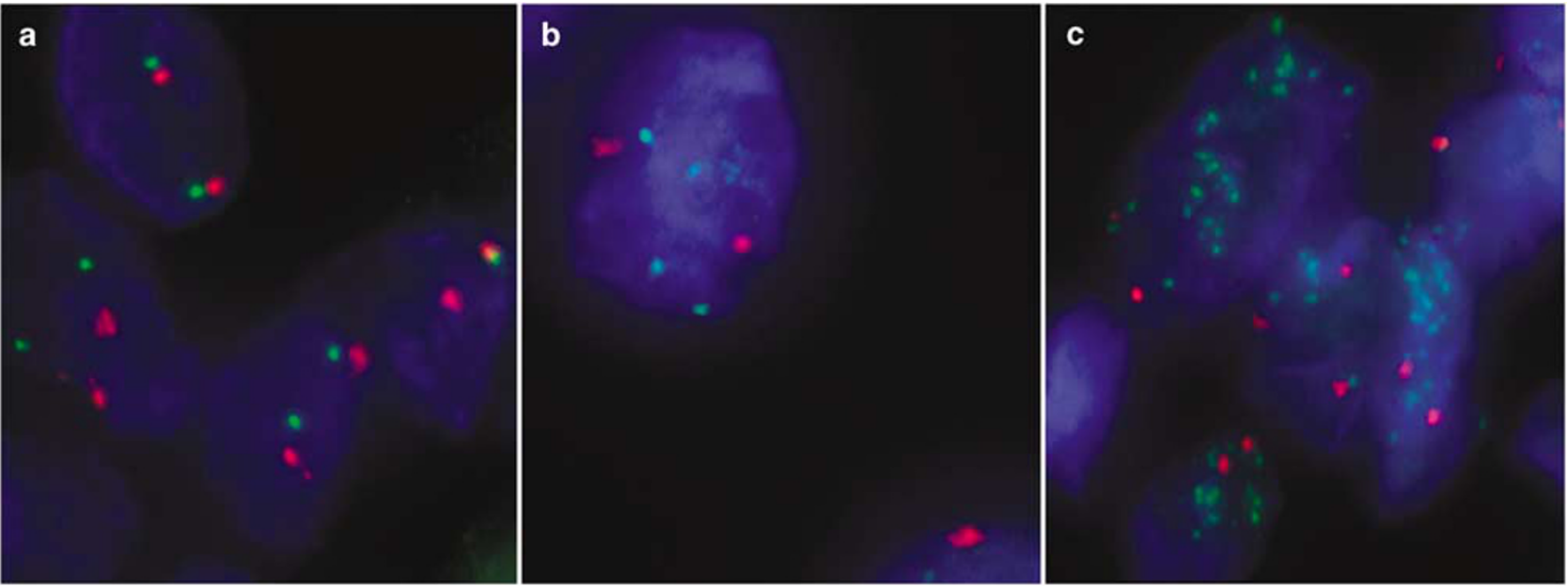

Figure $2 M D M 2$ amplification present in inflammatory well-differentiated liposarcoma/atypical lipomatous tumor (WDLS/ALT) and lack of amplification in sclerosing mesenteritis: (a) absence of MDM2 amplification in sclerosing mesenteritis, (b) low-level MDM2 amplification in inflammatory WDLS/ALT, and (c) high-level MDM2 amplification in inflammatory WDLS/ALT (MDM2/CEP12 FISH assay: MDM2-green signals, CEP12-red signals, $\times 100)$.

whereas the average MDM2/CEP12 ratio of retroperitoneal fibrosis and sclerosing mesenteritis cases was 0.95 and 0.90 , respectively.

\section{Discussion}

The inflammatory subtype of well-differentiated liposarcoma is rare-reported to represent less than $2 \%$ of 525 liposarcomas reviewed in one large series. ${ }^{11}$ Inflammatory well-differentiated liposarcoma/atypical lipomatous tumor can mimic sclerosing mesenteritis and retroperitoneal fibrosis, both clinically and radiographically. The histologic diagnosis can be problematic when the inflammatory infiltrate obscures the atypical spindle cells.

Well-differentiated liposarcoma/atypical lipomatous tumor have been shown by cytogenetics to contain characteristic 12q13-15 amplification on giant marker and ring chromosomes resulting in the amplification of several genes including MDM2. MDM2 is an oncogene that is important in controlling the cell cycle by binding to TP5 3 and promoting its degradation and therefore is thought to be directly involved in the pathogenesis of various neoplasms, including well-differentiated liposarcoma/atypical lipomatous tumor. ${ }^{18}$ We have shown that detection of MDM2 amplification by FISH can be useful in distinguishing well-differentiated liposarcoma/atypical lipomatous tumor from its histologic mimics. ${ }^{19}$

MDM2 IHC was found to be very sensitive (100\%) and rather specific (81\%) for differentiating inflammatory well-differentiated liposarcoma/atypical lipomatous tumor from sclerosing mesenteritis and retroperitoneal fibrosis. Although rare cases of sclerosing mesenteritis and retroperitoneal fibrosis displayed weak $(1+)$ positivity for MDM2 by IHC, none showed moderate-to-strong expression $(2-3+)$, which was seen in over half of all the welldifferentiated liposarcoma/atypical lipomatous tumor (Figure 1). Therefore, moderate or strong MDM2 expression is specific $(100 \%)$ for welldifferentiated liposarcoma/atypical lipomatous tumor, whereas if less than $25 \%$ of the lesional cells express MDM2 by IHC $(1+)$, the specificity is reduced to $81 \%$. It is important to realize that when interpreting the MDM2 IHC stain, the lesions are predominantly composed of inflammatory tissue, so that in reality there are very few neoplastic cells present in one microscopic field; therefore, even in those cases with $3+$ positivity, most of the cells on the slide are negative. Further testing by FISH may be required to rule out rare examples of sclerosing mesenteritis or retroperitoneal fibrosis that are weakly MDM2 positive by IHC. MDM2 antibody is a nuclear stain; consequently, nonspecific cytoplasmic staining of plasma cells and fibroblasts should be interpreted as negative for MDM2 expression. Furthermore, nuclear staining found within histiocytes in a minority of cases should not be confused with positive MDM2 immunoexpression within lesional cells.

The MDM2 FISH assay is a specific $(100 \%)$ and relatively sensitive adjunctive tool for distinguishing inflammatory well-differentiated liposarcoma from sclerosing mesenteritis and retroperitoneal fibrosis. No cases of sclerosing mesenteritis or retroperitoneal fibrosis were amplified including the four cases with weak MDM2 immunoreactivity. The two cases of well-differentiated liposarcoma/ atypical lipomatous tumor that were not amplified by MDM2 FISH were weakly positive by IHC. Therefore, similar cases should be considered equivocal and additional tissue may be required for definitive diagnosis. Importantly, five cases of retroperitoneal fibrosis were interpreted as unsatisfactory using MDM2 FISH due to autofluorescence. 
Two out of five cases were subsequently found to have been fixed in B5 solution, due to the clinical suspicion of lymphoma. FISH cannot be reliably performed on B5-fixed material. Of the remaining three cases showing autofluorescence, all were fixed in formalin, and although the exact cause of autofluorescence in a small subset of cases remains uncertain, it could be attributed to the time of fixation, which has been a documented problem in the analysis of Her2/Neu FISH assays. ${ }^{20}$ Therefore, although MDM2 FISH assay was very useful in distinguishing between inflammatory well-differentiated liposarcoma/atypical lipomatous tumor and sclerosing mesenteritis or idiopathic retroperitoneal fibrosis in the majority of cases, technical issues resulting in noncontributory results due to autofluorescence may limit the usefulness of the assay in certain situations.

The diagnosis of inflammatory well-differentiated liposarcoma/atypical lipomatous tumor can be exceedingly difficult and can be confused with fibroinflammatory disorders due to a predominance of the inflammatory infiltrate. However, we have shown that adjunctive analytical tools such as IHC and FISH can be used to detect increased MDM2 expression or MDM2 amplification and can be employed to help differentiate these entities with very high sensitivity and specificity. MDM2 FISH is a very specific method $(100 \%)$, but is less sensitive $(88 \%)$, whereas MDM2 expression by IHC is very sensitive $(100 \%)$, but less specific (83\%). The positive predictive value of the MDM2 FISH assay is $100 \%$, whereas it is only $81 \%$ for MDM2 IHC. The negative predictive value of the MDM2 FISH assay is $90 \%$, whereas it is $100 \%$ for MDM2 IHC. Therefore, a positive initial screen of difficult cases with MDM2 IHC would require confirmation by FISH. However, lack of MDM2 expression by IHC would rule out the possibility of inflammatory well-differentiated liposarcoma/atypical lipomatous tumor.

\section{References}

1 Levy AD, Rimola J, Mehrotra AK, et al. From the archives of the AFIP: benign fibrous tumors and tumorlike lesions of the mesentery: radiologic-pathologic correlation. Radiographics 2006;26:245-264.

2 Reske M, Namiki H. Sclerosing mesenteritis. Report of two cases. Am J Clin Pathol 1975;64:661-667.

3 Kelly JK, Hwang WS. Idiopathic retractile (sclerosing) mesenteritis and its differential diagnosis. Am J Surg Pathol 1989;13:513-521.

4 Durst AL, Freund H, Rosenmann E, et al. Mesenteric panniculitis: review of the literature and presentation of cases. Surgery 1977;81:203-211.

5 Kipfer RE, Moertel CG, Dahlin DC. Mesenteric lipodystrophy. Ann Intern Med 1974;80:582-588.
6 Emory TS, Monihan JM, Carr NJ, et al. Sclerosing mesenteritis, mesenteric panniculitis and mesenteric lipodystrophy: a single entity? Am J Surg Pathol 1997;21:392-398.

7 Vaglio A, Salvarani C, Buzio C. Retroperitoneal fibrosis. Lancet 2006;367:241-251.

8 Mitchinson MJ. The pathology of idiopathic retroperitoneal fibrosis. J Clin Pathol 1970;23:681-689.

9 Weiss SW, Goldblum JR. Liposarcoma. Enzinger and Weiss's Soft Tissue Tumors Vol., 5th edn. Mosby: St Louis, 2008, pp 477-516.

10 Dei Tos AP, Pedeutour F. Atypical lipomatous tumour/ well differentiated liposarcoma. In: Fletcher CD, Unni KK, Mertens F (eds). World Health Organization Classification of Tumours Pathology \& Genetics of Tumours of Soft Tissue and Bone, vol. IARC Press: Lyon, 2002, pp 35-37.

11 Kraus MD, Guillou L, Fletcher CD. Well-differentiated inflammatory liposarcoma: an uncommon and easily overlooked variant of a common sarcoma. Am J Surg Pathol 1997;21:518-527.

12 Argani P, Facchetti F, Inghirami G, et al. Lymphocyterich well-differentiated liposarcoma: report of nine cases. Am J Surg Pathol 1997;21:884-895.

13 Karakousis CP, Dal Cin P, Turc-Carel C, et al. Chromosomal changes in soft-tissue sarcomas. A new diagnostic parameter. Arch Surg 1987;122: 1257-1260.

14 Dal Cin P, Kools P, Sciot R, et al. Cytogenetic and fluorescence in situ hybridization investigation of ring chromosomes characterizing a specific pathologic subgroup of adipose tissue tumors. Cancer Genet Cytogenet 1993;68:85-90.

15 Pedeutour F, Suijkerbuijk RF, Forus A, et al. Complex composition and co-amplification of SAS and MDM2 in ring and giant rod marker chromosomes in welldifferentiated liposarcoma. Genes Chromosomes Cancer 1994;10:85-94.

16 Binh MB, Sastre-Garau X, Guillou L, et al. MDM2 and CDK4 immunostainings are useful adjuncts in diagnosing well-differentiated and dedifferentiated liposarcoma subtypes: a comparative analysis of 559 soft tissue neoplasms with genetic data. Am J Surg Pathol 2005;29:1340-1347.

17 Sirvent N, Coindre JM, Maire G, et al. Detection of MDM2-CDK4 amplification by fluorescence in situ hybridization in 200 paraffin-embedded tumor samples: utility in diagnosing adipocytic lesions and comparison with immunohistochemistry and real-time PCR. Am J Surg Pathol 2007;31: 1476-1489.

18 Vargas DA, Takahashi S, Ronai Z. Mdm2: a regulator of cell growth and death. Adv Cancer Res 2003;89: $1-34$.

19 Weaver J, Downs-Kelly E, Goldblum JR, et al. Fluorescence in situ hybridization for MDM2 gene amplification as a diagnostic tool in lipomatous neoplasms. Mod Pathol 2008;21:943-949.

20 Wolff AC, Hammond ME, Schwartz JN, et al. American Society of Clinical Oncology/College of American Pathologists guideline recommendations for human epidermal growth factor receptor 2 testing in breast cancer. J Clin Oncol 2007;25:118-145. 